\title{
Decentralized Model Predictive Control for Automated and Connected Electric Vehicles at Signal-free Intersections
}

\author{
Xiao Pan, Boli Chen, Li Dai, Stelios Timotheou, and Simos A. Evangelou
}

\begin{abstract}
The development of connected and automated vehicles (CAVs) enables improvements in the safety, smoothness, and energy efficiency of the road transportation systems. This paper addresses the problem of optimally controlling batteryelectric CAVs crossing an unsignalized intersection subject to a first-in-first-out crossing policy. The optimal velocity trajectory of each vehicle that minimizes the average energy consumption and travel time, is found by a decentralized model predictive control (DMPC) method via a convex modeling framework so as to ensure computational efficiency and the optimality of the solution. Numerical examples and comparisons with a centralized control counterpart demonstrate the effectiveness of the proposed decentralized coordination scheme and the trade-off between energy consumption and travel time. Further investigation into the size of the sampling interval is also provided in order to show the validity of the method in practice.
\end{abstract}

\section{INTRODUCTION}

Road congestion and strained transportation networks are persistent concerns associated with rapid urbanization and the increasing number of vehicles on the road. With the advancement of connected and autonomous vehicle (CAV) technologies, significant research efforts have been made on cooperative vehicle management, which has the potential to alleviate traffic congestion and therefore improve throughput and energy efficiency. This technique is particularly relevant to road traffic management at intersections, where different traffic flows merge together. Compared to the traditional traffic light control methods, autonomous (signal-free) intersection control is an innovative technology that relies only on the advanced vehicular communication system and therefore it could improve intersection throughput with a minimum investment on the infrastructure [1], [2], [3].

A comprehensive overview of recent advancements in centralized and decentralized autonomous intersection control approaches is presented in [4], [5]. The core of the centralized methods is a central intersection controller (IC), which

This work has been supported by the EPSRC Grant EP/N022262/1 and partially funded by the European Union's Horizon 2020 research and innovation programme under grant agreement No 739551 (KIOS CoE) and the Government of the Republic of Cyprus through the Directorate General for European Programmes, Coordination and Development.

X. Pan and S. A. Evangelou are with the Dept. of Electrical and Electronic Engineering, Imperial College London, London, UK (xiao.pan17@ic.ac.uk, s.evangeloudic.ac.uk).

B. Chen is with the Dept. of Electronic and Electrical Engineering, University College London, London, UK (boli.chen@ucl.ac.uk).

L. Dai is with the School of Automation, Beijing Institute of Technology, Beijing, China (li.dai@bit.edu.cn)

S. Timotheou is with the Dept. of Electrical and Computer Engineering and the KIOS Research and Innovation Center of Excellence, University of Cyprus, Cyprus (timotheou.stelios@ucy.ac.cy). determines the optimal velocity trajectories of all CAVs approaching the intersection with the aim of minimizing average energy consumption and travel time [6], [7], [8]. The trade-off between travel time and energy consumption minimization is investigated in [9], where the problem is addressed by a hierarchical and convex optimization method. More recently, in [10], [11], the problem is recast by considering electric CAVs and various friction losses, and a convex reformulation approach is proposed to alleviate the overall computational complexity. The development of the more realistic modeling framework by the inclusion of explicit powertrain and vehicle dynamics models is shown to lead to a more accurate energy consumption evaluation, as compared to existing work that employs conventional simplistic lossless vehicles models.

Despite the efficiency of the convex optimization, the centralized scheme still has disadvantages in computational efficiency and resilience against the failure of the central controller, as the whole system relies on the central controller. A more practical alternative is the decentralized control framework, where each CAV is controlled by its local controller in conjunction with the communication and sensing systems [12], [13], [14]. In [12], a sequential optimal control approach is proposed to maximize the throughput, along with a computational efficient scheduling method. An analytic optimization method is proposed in [13] where the energy-optimal trajectory of each CAV is found individually by Pontryagin's minimum principle. Multi-agent Markov decision processes are utilized in [14] to model the sequential movement of CAVs such that each velocity trajectory can be optimally determined by a reinforcement learning method. More computationally efficient methods are also designed based on heuristic control strategies [15], [16], which however lack optimality guarantees.

The present paper addresses the autonomous intersection crossing problem by a decentralized method, where the speed trajectory of each CAV is sequentially optimized by a decentralized model predictive control (DMPC) method targeting minimum travel time and energy consumption. Compared to a centralized MPC setup, where a global optimal control problem is solved with respect to the overall dynamics and constraints, the DMPC enables the problem to be decoupled into local (of smaller size) MPC problems. These local MPC systems cooperatively control the large-scale system by exchanging information through communication channels. As such, online implementation is allowed with superior optimality as compared to other non-optimization methods. The modeling framework with electric CAVs proposed in 
prior work [11] of the authors is utilized in the present work. The optimization problem is formulated in a convex form under the space domain rather than the commonly used time domain, which enables a convenient deployment of DMPC, as the boundary conditions (in terms of the travel distance) are fixed. The optimality of the DMPC solution is validated by numerical investigation into the impact of the prediction horizon length and by a comparison with the optimal solutions obtained by a centralized method [11] under the same modeling framework. The computational efficiency of the proposed decentralized control scheme is examined by evaluating the running time for a single MPC at each distance sample, and the results provide insight into the setting of the sampling distance interval and also show the practicality of the proposed methodology. The contributions of the present work can be summarized as follows: 1) a first in the literature combination of decentralized control method for the autonomous intersection problem with a realistic vehicle modeling framework, and 2) that innovatively also combines decentralization with model predictive control in the space domain, which avoids the difficulty of solving the end-time free optimization in the time domain.

The rest of this paper is organized as follows. Section 1 introduces the convex modeling framework of an autonomous intersection crossing problem as well as the electric powertrain model of the CAV. The convex autonomous intersection crossing problem is formulated as a decentralized MPC problem in Section III. Simulation results are presented and discussed in Section [V] Finally, concluding remarks are given in Section $\mathrm{V}$

\section{Problem Statement}

\section{A. Intersection Model}

A signal-free traffic intersection that is formed by two perpendicular roads with two lanes per road and a group of homogeneous battery electric CAVs is considered. This paper deals with a decentralized control scheme for the cooperative intersection crossing of all CAVs. The schematic of the intersection is shown in Fig. 1 As it can be seen, vehicles approaching the intersection will first enter a control zone (CZ) of length $L$. The intersection also has a coordinator that facilitates exchange of information among the CAVs inside the $\mathrm{CZ}$, therefore, $L$ is determined by the communication range capability of the CAVs and the coordinator. Note that the intersection coordinator is only used to streamline the communication network for the decentralized control scheme, which will be specified in Section III] and it is not involved in making control decisions. The centre of the intersection is called the Merging Zone (MZ), where vehicles merge from different directions, and therefore where lateral collision may occur. The area of the MZ is considered as a square of side $S$, with $S<L$, as the physical length of the MZ is usually much smaller than the sensing range of wireless communication devices. For simplicity, it is assumed that the roads are flat and all CAVs maintain their initial direction after exiting the MZ. The control target is to minimize the average electric energy consumption and travel

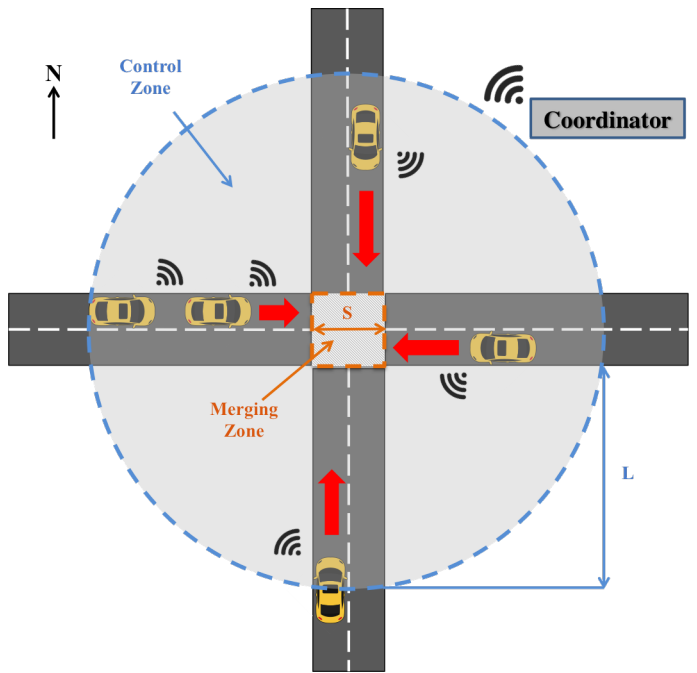

Fig. 1. The schematic of autonomous intersection with connected and autonomous vehicles.

time by finding in a decentralized manner the optimal speed trajectory for each vehicle from the entry of the $\mathrm{CZ}$ to the exit point of the MZ.

Let us denote $N(t) \in \mathbb{N}_{>0}$ the total number of CAVs within the $\mathrm{CZ}$ at a given time $t \in \mathbb{R}_{>0}$ and $\mathcal{N}(t)=\{1,2, \ldots, N(t)\}$ the set to designate the order in which the vehicles enter the CZ. The sequence $\mathcal{N}(t)$ is recorded by the coordinator and will later be used to generate the corresponding information set exchanged with each CAV that follows a First-In-FirstOut (FIFO) policy (all CAVs enter and leave the MZ in the same order they arrive at the $\mathrm{CZ}$ ). The following definitions are given for the further discussion of collision avoidance constraints. Given an arbitrary CAV $h$, any CAV $i \in \mathcal{N}(t)$ arriving at the $\mathrm{CZ}$ later than the $h$ th $\mathrm{CAV}(h<i)$ can be categorized into one of the following subsets of $\mathcal{N}$ based on its physical location inside the $\mathrm{CZ}: 1) \mathcal{C}_{h}$ collects vehicles traveling in the same direction as the $i$ th vehicle; 2) $\mathcal{O}_{h}$ collects vehicles traveling in the opposite direction to the $i$ th vehicle; 3) $\mathcal{L}_{h}$ collects vehicles traveling in the perpendicular directions to the $i$ th vehicle.

In this paper, the autonomous intersection crossing problem is modeled in the space domain for two reasons: (a) the free end-time optimization problem in the time domain is avoided and (b) the problem can be formulated in convex form [11]. Let $s$ denote the variable of traveled distance and $v_{i}(s)$ denote the velocity of the $i$ th vehicle. The transformation from time to space domain is achieved by changing the independent variable $t$ to $s$ via $\frac{d}{d s}=\frac{1}{v_{i}} \frac{d}{d t}$. Thus, in the space domain, the travel distance for each CAV is constant at $L+S$ and the travel time of each CAV can be easily obtained as a state variable, $\frac{d}{d s} t_{i}=\frac{1}{v_{i}}$, and therefore, the required travel time of each CAV to cross the intersection is:

$$
J_{t, i}=t_{i}(L+S)-t_{i}(0),
$$

where $t_{i}(0)$ is the arrival time of CAV $i$ at the CZ.

The electric energy cost of each CAV, $J_{b, i}$, is evaluated based on the static efficiency map of the tank-to-wheel energy path [11], given in Fig. 2, including the efficiency 
of the electric motor, converter and fixed gear. Although the

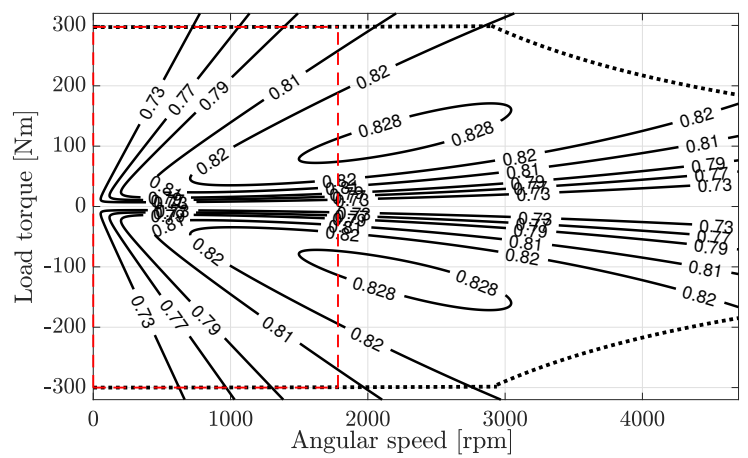

Fig. 2. Efficiency map of the electric motor (positive torque indicates battery discharging and negative torque represents battery charging) and operational bounds (dotted lines) for the reversible motor. The area surrounded by red dashed lines denotes the operational region for the feasible vehicle speed specified by $4 \mathrm{e}$, with $v_{\min }$ and $v_{\max }$ defined in Table []

motor torque is constrained by speed dependent limits, the motor torque limits $T_{\max }$ and $T_{\min }$ are constant until the angular speed, $\omega_{i}(s)$, reaches approximately $2800 \mathrm{rpm}$, which corresponds to a forward speed at $25 \mathrm{~m} / \mathrm{s}$, as shown in Fig. 2 . However, it is reasonable to impose a much lower speed limit for vehicles approaching an intersection. Considering the speed limit $v_{\max }=15 \mathrm{~m} / \mathrm{s}$ given in Table I] constant motor toque limits can be applied, $T_{i} \in\left[T_{\min }, T_{\max }\right], \forall i \in \mathcal{N}$, where $T_{i}$ is the torque of the $i$ th $\mathrm{CAV}$, and $T_{\min }=-300 \mathrm{Nm}$, $T_{\max }=300 \mathrm{Nm}$.

The battery output power can be represented by a quadratic function of the powertrain traction force and driving speed that are proportional to the motor torque and angular speed, respectively, as follows:,

$$
P_{b, i}=b_{1} F_{t, i}^{2} v_{i}+b_{2} F_{t, i} v_{i}+b_{3} v_{i}
$$

where $F_{t, i}(s)=\frac{g_{r}}{r_{w}} T_{i}(s), v_{i}(s)=\frac{r_{w}}{g_{r}} \omega_{i}(s), g_{r}$ and $r_{w}$ are the transmission gear ratio and the wheel radius, respectively, and $b_{1}, b_{2}$ and $b_{3}$ are the fitting parameters for the map given in Fig. 2. In view of (2), the battery energy usage in

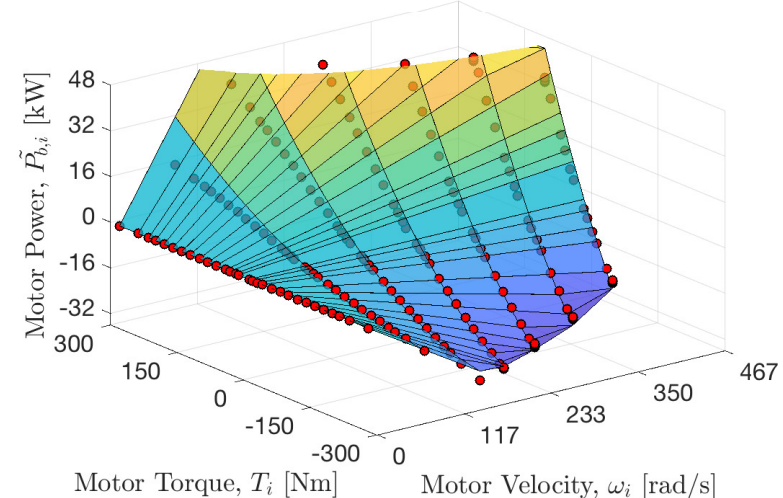

Fig. 3. Nonlinear regression of the battery output power data (red dots, calculated based on the efficiency map shown in Fig. 2 by using the approximated model 22 with an R-square fit of $99.53 \%$. the space domain of each $\mathrm{CAV}$ is:

$$
J_{b, i}=\int_{0}^{L+S} \frac{P_{b, i}(s)}{v_{i}(s)} d s=\int_{0}^{L+S}\left(b_{1} F_{t, i}^{2}(s)+b_{2} F_{t, i}(s)+b_{3}\right) d s .
$$

The main characteristic parameters of each vehicle model are summarized in Table []

TABLE I

Electric Vehicle Model Parameters

\begin{tabular}{lcl}
\hline \hline symbol & value & description \\
\hline$m$ & $1200 \mathrm{~kg}$ & vehicle mass \\
$r_{w}$ & $0.3 \mathrm{~m}$ & wheel radius \\
$g_{r}$ & 3.5 & transmission gear ratio \\
$f_{r}$ & 0.01 & rolling resistance coefficient \\
$f_{d}$ & 0.47 & air drag resistance coefficient \\
$v_{\min }$ & $0.1 \mathrm{~m} / \mathrm{s}$ & minimum velocity \\
$v_{\max }$ & $15 \mathrm{~m} / \mathrm{s}$ & maximum velocity \\
$b_{1}, b_{2}, b_{3}$ & $7.15 \mathrm{e}-4,0.8842,5.35$ & powertrain fitting parameters \\
\hline \hline
\end{tabular}

Taking into account the FIFO policy and the objective of minimizing the electric energy (3) and travel time (1), the global optimization problem can be formulated as follows [11] (this is instrumental for introducing the DMPC algorithm in the upcoming Section):

$$
\begin{aligned}
& \min _{\mathbf{u}} \quad J=W_{1} \sum_{i=1}^{N} J_{t, i}+W_{2} \sum_{i=1}^{N} J_{b, i} \\
& \text { s.t. : }(\forall s \in[0, L+S], i \in \mathcal{N}) \\
& \frac{d}{d s} E_{i}(s)=F_{t, i}(s)+F_{b, i}(s)-F_{r}-\frac{2 f_{d}}{m} E_{i}(s), \\
& \frac{d}{d s} t_{i}(s)=\zeta_{i}(s), \\
& \zeta_{i}(s) \geq \frac{1}{\sqrt{2 E_{i}(s) / m}}, \\
& \frac{1}{2} m v_{\min }^{2} \leq E_{i}(s) \leq \frac{1}{2} m v_{\max }^{2} \\
& \frac{g_{r}}{r_{w}} T_{\min } \leq F_{t, i}(s) \leq \frac{g_{r}}{r_{w}} T_{\max }, \\
& m a_{\min }-\frac{g_{r}}{r_{w}} T_{\min } \leq F_{b, i}(s) \leq 0, \\
& t_{i}(s)-t_{h}(s) \geq \beta(s), \forall i \in \mathcal{C}_{h}, \\
& t_{i}(L) \geq t_{h}(L+S), \forall i \in \mathcal{L}_{h}, \\
& t_{i}(L)>t_{h}(L), t_{i}(L+S)>t_{h}(L+S), \forall i \in \mathcal{O}_{h}, \\
& E_{i}(L+S)=\frac{1}{2} m \bar{v}^{2},
\end{aligned}
$$

where

$$
\begin{gathered}
\mathbf{u}=\left[F_{t, 1}(s), F_{t, 2}(s), \cdots, F_{t, N}(s), F_{b, 1}(s), F_{b, 2}(s),\right. \\
\left.\cdots, F_{b, N}(s), \zeta_{1}(s), \zeta_{2}(s), \cdots, \zeta_{N}(s)\right]^{\top} \in \mathbb{R}^{3 N},
\end{gathered}
$$

$W_{1} \geq 0$ and $W_{2} \geq 0$ are weighting factors, $m$ is the vehicle mass, $F_{b, i}(s)$ is the mechanical braking force, $F_{r}=f_{r} m g$ is the rolling resistance force, and $f_{r}$ and $f_{d}$ are the coefficients of rolling and air drag resistances, respectively. Instead of using the velocity $v_{i}$ as a state, the longitudinal dynamics of each CAV are modeled in 4b with respect to the kinetic energy, $E_{i}(s)=\frac{1}{2} m v_{i}^{2}(s)$, to cancel the nonlinearity due to 
the air drag. The dynamics of $t_{i}$ are modeled by 4c and (4d), which relax the original nonlinear differential equation into a linear differential equation and a convex constraint of the auxiliary control variable $\zeta_{i}(s)$. It is worth noting that the validity of the final solution relies on the tightness of $(4 \mathrm{~d})$. A rigorous investigation of the tightness of (4d) is beyond the scope of the present article and will be addressed in future work, however, for all the practical scenarios of interest in the present work it has been found that the present formulation yields a tight solution, which is considered adequate for the present purposes; see also [11]. The kinetic energy of each CAV is bounded by (4e), where without loss of generality $v_{\text {min }}$ is set to a sufficiently small positive constant to avoid singularity issues that would appear in $4 c$ when $v_{i}=0$, and $v_{\max }$ is determined based on the infrastructure constraints and traffic regulations [9]. Vehicle driving/braking forces $F_{t, i}(s), F_{b, i}(s)$ are constrained by (4f) and (4g). $a_{\min }=$ $-6.5 \mathrm{~m} / \mathrm{s}^{2}$ is the maximum available deceleration during emergency braking subject to tire friction limits. As it can be noticed in (4g), $a_{\text {min }}$ is achieved by a combination of regenerative and mechanical braking.

The rear-end collision avoidance constraint is given in (4h). The right hand side of the inequality represents the time-to-collision (TTC),

$$
\beta(s)=\frac{v_{i}(s)-v_{h}(s)}{\left|a_{\min }\right|}=\frac{\sqrt{2 E_{i}(s) / m}-\sqrt{2 E_{h}(s) / m}}{\left|a_{\min }\right|} .
$$

To convexify (4h), the nonlinear term $\sqrt{2 E_{i}(s) / m}$ is approximated by $f^{*}\left(E_{i}(s)\right)=a_{0}^{*}+a_{1}^{*} E_{i}(s)$, where $a_{0}^{*}$ and $a_{1}^{*}$ are obtained through a constrained least-squares optimization:

$$
\begin{array}{ll} 
& \min _{a_{0}, a_{1}}\left\|f\left(E_{i}\right)-\sqrt{2 E_{i} / m}\right\|_{2} \\
\text { s.t.: } & f\left(E_{i}\right)-\sqrt{2 E_{i} / m} \geq 0, \\
& E_{i} \in\left[\frac{1}{2} m v_{\min }^{2}, \frac{1}{2} m v_{\max }^{2}\right],
\end{array}
$$

which is formed to maximize feasibility while preserving convexity. The problem has a unique solution $f^{*}\left(E_{i}(s)\right)$ that is tangential to $\sqrt{2 E_{i}(s) / m}$, as shown in Fig. 4. As it can be seen, the TTC constraint $4 \mathrm{~h}$ is active only when $v_{i}(s)>$ $v_{h}(s)$ while aiming to reduce the inter-vehicular distance for increased road capacity. Lateral collisions are avoided by

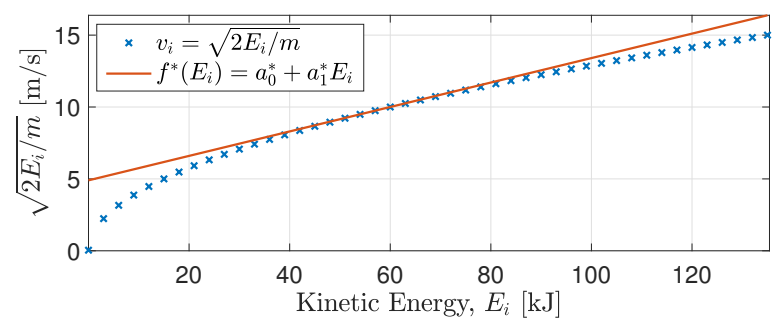

Fig. 4. Linearly approximated relationship between kinetic energy and velocity with $\mathrm{R}$-square fit of $92.27 \%$.

(4i), which guarantees that the $i$ th vehicle enters the MZ only after the $h$ th vehicle leaves the MZ. For any CAV $i \in \mathcal{O}_{h}$, there is no interference between CAVs $h$ and $i$ inside the $\mathrm{CZ}$. Hence, only (4j) is required to ensure the FIFO policy.
Finally, the optimization problem is completed by the terminal speed condition $4 \mathrm{k}$, where $\bar{v}$ can be chosen arbitrarily within $\left[v_{\min }, v_{\max }\right]$. Note that the same terminal speed for all CAVs is imposed only to allow solutions in different scenarios to be easily compared. It is straightforward to relax 4k with non-uniform terminal speeds.

The following assumptions are also needed.

Assumption 1: All the vehicle information (e.g., position, velocity, acceleration) can be measured precisely, and the data can be transferred between each CAV and the coordinator without errors and delays.

Assumption 2: The entry times of all CAVs at the CZ are different, such that $t_{i}(0) \neq t_{h}(0), i \neq h, i, h \in\{1,2, \cdots, N\}$.

Assumption 3: For each CAV $i$, constraints (4e), 4f], 4g) and (4h) are inactive at $t_{i}(0)$.

Assumption 2 is intended to validate the FIFO priority model. Under this assumption, the FIFO policy can be guaranteed when (4h), 4i), 4j) are met. Assumption 3 is needed to ensure feasible initial states and initial control inputs.

\section{DECENTRALIZED MPC FORMULATION}

It is straightforward to solve the optimization problem (4) using a centralized control scheme, which yields speed trajectories of all the CAVs and the associated control actions for the whole mission [11]. Nevertheless, it is challenging to implement centralized methods due to the limited computational resources in reality and the uncertain arrival time and speed of the second or later CAVs in the sequence $\mathcal{N}$. This section reformulates (4) as a DMPC problem, which finds a balance between optimality and computational efficiency.

Let us denote $N_{p} \in \mathbb{N}_{>0}$ the prediction horizon. The vehicle dynamics $4 \mathrm{4b}-(4 \mathrm{c})$ can be rewritten in a discrete state-space form with a sampling distance interval $\Delta s \in \mathbb{R}_{>0}$ :

$$
\left[\begin{array}{c}
E_{i}(k+1) \\
t_{i}(k+1)
\end{array}\right]=A_{d}\left[\begin{array}{c}
E_{i}(k) \\
t_{i}(k)
\end{array}\right]+B_{d}\left[\begin{array}{c}
F_{t, i}(k) \\
F_{b, i}(k) \\
\zeta_{i}(k)
\end{array}\right]+\Delta s\left[\begin{array}{c}
-F_{r} \\
0
\end{array}\right]
$$

where,

$$
A_{d}=\left[\begin{array}{cc}
1-\frac{2 f_{d}}{m} \Delta s & 0 \\
0 & 1
\end{array}\right], \quad B_{d}=\left[\begin{array}{ccc}
\Delta s & \Delta s & 0 \\
0 & 0 & \Delta s
\end{array}\right] .
$$

Without loss of generality, it is assumed that $L+S=\alpha_{1} \Delta s$ and $L=\alpha_{2} \Delta s, \alpha_{1}, \alpha_{2} \in \mathbb{N}_{>0}, \alpha_{1}>\alpha_{2}$.

In the decentralized framework, the coordinator assigns a unique identity to each $\mathrm{CAV}$ when the vehicle enters the $\mathrm{CZ}$ of the intersection based on the vehicle's arrival time and entering direction. Let $M(t)$ be the cumulative number of CAVs that have entered the $\mathrm{CZ}$ by time $t$. The identity that the coordinator assigns to vehicle $i$ that arrives at the $\mathrm{CZ}$ is defined as $\left(\gamma_{i}, d_{i}\right)$, where $\gamma_{i}=M(t)+1$, and $d_{i} \in\{1,2,3\}$ is an integer based on a one-to-one mapping with respect to $\left\{\mathcal{C}_{h}, \mathcal{O}_{h}, \mathcal{L}_{h}\right\}$ that indicates the relationship between the $i$ th $\mathrm{CAV}$ and the vehicle $h$ entering the $\mathrm{CZ}$ at an earlier time. At any step $k$, the coordinator requests from each 
CAV the optimal state sequence $\mathbf{x}_{\gamma_{i}}(k)=\left[\mathbf{E}_{\gamma_{i}}(k), \mathbf{t}_{\gamma_{i}}(k)\right] \in$ $\mathbb{R}^{\left(N_{p}+1\right) \times 2}$ within the receding horizon $N_{p}$ :

$$
\begin{aligned}
\mathbf{E}_{\gamma_{i}}(k) & =\left[E_{\gamma_{i}}(k \mid k), E_{\gamma_{i}}(k+1 \mid k), \ldots, E_{\gamma_{i}}\left(k+N_{p} \mid k\right)\right]^{\top}, \\
\mathbf{t}_{\gamma_{i}}(k) & =\left[t_{\gamma_{i}}(k \mid k), t_{\gamma_{i}}(k+1 \mid k), \ldots, t_{\gamma_{i}}\left(k+N_{p} \mid k\right)\right]^{\top} .
\end{aligned}
$$

As such for each CAV $i$ at step $k$, the coordinator can generate an information set:

$$
\mathcal{I}_{\gamma_{i}}(k)=\left[\mathbf{x}_{\gamma_{i}}(0), \mathbf{x}_{\gamma_{i}}(1), \cdots, \mathbf{x}_{\gamma_{i}}(k)\right] \in \mathbb{R}^{\left(N_{p}+1\right) \times 2 k} .
$$

Then, based on $\left(\gamma_{i}, d_{i}\right)$, the coordinator exchanges the information set $\mathcal{I}_{\gamma_{i}}(k)$ with the associated vehicles (see below) to enable collision avoidance constraints to be established for each local MPC. The information any CAV $i$ requires is:

1) no information is required, if $\gamma_{i}=1$,

2) $\mathcal{I}_{\gamma_{i-1}}(k)$, if $\gamma_{i} \in \mathcal{C}_{\gamma_{i-1}}$,

3) $\mathcal{I}_{\gamma_{i-1}}(k)$ and $\mathcal{I}_{\gamma_{h}}(k)$ with $\gamma_{h}<\gamma_{i}$, where $h$ stands for the CAV immediately ahead of CAV $i$, if $\gamma_{i} \notin \mathcal{C}_{\gamma_{i-1}}$.

The objective function in the DMPC framework for vehicle $i$ is designed based on 4a):

$$
\begin{gathered}
\tilde{J}_{\gamma_{i}, k}=\sum_{j=0}^{N_{p}-1}\left[W_{1} P_{b, \gamma_{i}}(k+j \mid k)+W_{2} \zeta_{\gamma_{i}}(k+j \mid k)\right] d s \\
+W_{3}\left(E_{\gamma_{i}}\left(k+N_{p} \mid k\right)-\frac{m}{2} \bar{v}^{2}\right)^{2}, k=0,1, \cdots, \alpha_{1} .
\end{gathered}
$$

where the third term in 10 is a terminal cost. It is used to penalize the kinetic energy difference between $E_{\gamma_{i}}\left(k+N_{p} \mid k\right)$ and the desired exit condition $\frac{1}{2} m \bar{v}^{2}$ of the $\mathrm{MZ}$, to avoid the suboptimal solution where the final speed tends to reach $v_{\gamma_{i}}\left(k+N_{p} \mid k\right)=v_{\min }$.

The constraints $4 \mathrm{~d}-4 \mathrm{~g}$ ) can be immediately incorporated into the DMPC framework as they are imposed on a single vehicle. For CAV $i$ at each step, it follows that:

$$
\begin{aligned}
& \zeta_{\gamma_{i}}(k+j \mid k) \geq \frac{1}{\sqrt{2 E_{\gamma_{i}}(k+j \mid k) / m}}, \\
& \frac{1}{2} m v_{\min }^{2} \leq E_{\gamma_{i}}(k+j+1 \mid k) \leq \frac{1}{2} m v_{\max }^{2} \\
& \frac{g_{r}}{r_{w}} T_{\min } \leq F_{t, \gamma_{i}}(k+j \mid k) \leq \frac{g_{r}}{r_{w}} T_{\max }, \\
& m a_{\min }-\frac{g_{r}}{r_{w}} T_{\min } \leq F_{b, \gamma_{i}}(k+j \mid k) \leq 0,
\end{aligned}
$$

where $j=0,1, \cdots, N_{p}-1$.

In terms of the collision avoidance constraints 4h- 4j that involve more than one vehicles, the information set $\mathcal{I}_{\gamma_{i}}$ received from other vehicles (via the coordinator) is invoked. For any CAV $i$, if there exists a vehicle $h, \gamma_{h}<\gamma_{i}$ that is immediately ahead of CAV $i$, such that $\gamma_{i} \in \mathcal{C}_{\gamma_{h}}$, the following rear-end collision constraint is incorporated into the DMPC of vehicle $i$ :

$$
t_{\gamma_{i}}(k+j+1 \mid k)-t_{\gamma_{h}}(k+j+1 \mid k) \geq \beta(k+j+1 \mid k),
$$

where $j=0,1, \cdots, N_{p}-1$, and $t_{\underline{\gamma}_{h}}(k+j+1 \mid k)$ is the historical information stored in $\mathcal{I}_{\gamma_{h}}(\bar{k})$, where $\bar{k}>k$ is the corresponding distance step of CAV $h$ when the $i$ th CAV is at step $k$, that is $t_{\gamma_{h}}(\bar{k} \Delta s)=t_{\gamma_{i}}(k \Delta s)$.
In the DMPC framework, the lateral collision constraint for any CAV $i$ is required only when $\gamma_{i} \in \mathcal{L}_{\gamma_{i-1}}$, and the constraint is not active until the receding space window of the $\gamma_{i}$ th vehicle reaches the entry of MZ. However, the information of $t_{\gamma_{i-1}}(L+S)$ is not available from $\mathcal{I}_{\gamma_{i-1}}$ until the space window of the $\gamma_{i-1}$ th CAV reaches the exit of MZ. In this context, the reformatted lateral collision avoidance constraint is given by:

$$
t_{\gamma_{i}}(k+j+1 \mid k) \geq \hat{t}_{\gamma_{i-1}}^{\mathcal{L}}(L+S), \quad j \in \mathbb{N}_{\left[0, N_{p}-1\right]},
$$

where $k+j+1=\alpha_{2}$ with $\alpha_{2} \in \mathbb{N}_{\left[k, k+N_{p}\right]}$. The exit time of the $\gamma_{i-1}$ th CAV of MZ, $\hat{t}_{\gamma_{i-1}}(L+S)$, is estimated by

$$
\hat{t}_{\gamma_{i-1}}^{\mathcal{L}}(L+S)=\left\{\begin{array}{c}
t_{\gamma_{i-1}}(\bar{k}+l+1 \mid \bar{k}), \text { if } l<N_{p}, \bar{k}+l+1=\alpha_{1}, \\
t_{\gamma_{i-1}}\left(\bar{k}+N_{p} \mid \bar{k}\right)+\frac{L+S-\left(\bar{k}+N_{p}\right) \Delta s}{v_{\gamma_{i-1}}\left(\bar{k}+N_{p} \mid \bar{k}\right)}, \\
\text { if } \bar{k}+N_{p}<\alpha_{1},
\end{array}\right.
$$

where $l \in \mathbb{N}_{\left[0, N_{p}-1\right]}$, and $\bar{k}$, similarly to the earlier definition, stands for the associated step of the $\gamma_{i-1}$ th CAV such that $t_{\gamma_{i-1}}(\bar{k} \Delta s)=t_{\gamma_{i}}(k \Delta s)$. In the first case in (14), $\hat{t}_{\gamma_{i-1}}^{\mathcal{L}}(L+$ $S)$ is obtained straightforwardly in the first case, as the terminal condition is included in the space window of the $\gamma_{i-1}$ th vehicle. In the second case in (14), the terminal time $\hat{t}_{\gamma_{i-1}}^{\mathcal{L}}(L+S)$ is unavailable from the information received by the $\gamma_{i}$ th CAV; instead, it is estimated by assuming that the $\gamma_{i-1}$ th CAV will finish the rest of the mission at its terminal speed of the present horizon.

The constraints 4j] are needed when vehicle $\gamma_{i} \in \mathcal{O}_{\gamma_{i-1}}$. By analogy to the lateral collision avoidance constraint, (4j) is rewritten as follows:

$$
\begin{aligned}
& t_{\gamma_{i}}(k+j+1 \mid k) \geq \hat{t}_{\gamma_{i-1}}^{\mathcal{O}}(L), k+j+1=\alpha_{2} \\
& t_{\gamma_{i}}(k+l+1 \mid k) \geq \hat{t}_{\gamma_{i-1}}^{\mathcal{O}}(L+S), k+l+1=\alpha_{1}
\end{aligned}
$$

where $j, l \in \mathbb{N}_{\left[0, N_{p}-1\right]}$, while $\hat{t}_{\gamma_{i-1}}^{\mathcal{O}}(L)$ and $\hat{t}_{\gamma_{i-1}}^{\mathcal{O}}(L+S)$ can be estimated using the same approach given in (14).

Finally, the terminal speed constraint (4k) triggers when the position of the exit of the MZ falls into the prediction horizon of vehicle $i$, such that $\alpha_{1} \in \mathbb{N}_{\left[k, k+N_{p}\right]}$. With MPC, the receding window can surpass the exit of the MZ, that is $k+N_{p} \geq \alpha_{1}$. To ensure that the terminal condition is fulfilled under this circumstance, when the horizon surpasses $\alpha_{1}$, the speed for that part of the horizon is enforced to the desired terminal speed, such that:

$$
E_{\gamma_{i}}(k+j+1 \mid k)=\frac{1}{2} m \bar{v}^{2}, \text { if } k+N_{p} \geq \alpha_{1}
$$

where $j=\alpha_{1}-k-1, \alpha_{1}-k, \ldots, N_{p}-1$.

The DMPC framework of the autonomous intersection crossing control problem (4) for each CAV $i \in \mathcal{N}(t)$ can finally be formulated as:

$$
\begin{aligned}
\min _{\mathbf{u}_{\gamma_{i}}} & \tilde{J}_{\gamma_{i}, k}\left(\mathbf{x}_{\gamma_{i}}(k), \mathbf{u}_{\gamma_{i}}(k)\right), \\
\text { s.t. : } & (7),(11,13,15,16,15) \\
& x_{\gamma_{i}}(k \mid k)=x_{\gamma_{i}}(k) \\
\text { given : } & x_{\gamma_{i}}(0) .
\end{aligned}
$$


where $\mathbf{x}_{\gamma_{i}}(k) \in \mathbb{R}^{\left(N_{p}+1\right) \times 3}, \mathbf{u}_{\gamma_{i}}(k) \in \mathbb{R}^{N_{p} \times 3}, k=0,1, \ldots, \alpha_{1}$, $x_{\gamma_{i}}(k)$ in $(17 \mathrm{c})$ is the actual state measured at step $k$, and $x_{\gamma_{i}}(0)$ in $(17 \mathrm{~d})$ is the initial state (entry state at CZ) satisfying Assumptions 2,3 .

Remark 1: The choice of the sampling distance interval is important for the problem, and its tuning is subject to the typical trade-off between accuracy and computational complexity, which influences the setup of the DMPC. In practice, due to the inevitable delays in computation and communication, the information $\mathcal{I}_{\gamma_{i-1}}(\bar{k})$ required for constraints (13) and (15) may not be available at step $k$ of the $\gamma_{i}$ th CAV. In such case, the $\gamma_{i}$ th vehicle can use the latest data of the $\gamma_{i-1}$ th vehicle contained in $\mathcal{I}_{\gamma_{i-1}}$, and then, the arrival time of the $\gamma_{i-1}$ th CAV at the entry and exit of the MZ $\hat{t}_{\gamma_{i-1}}^{\mathcal{L}}(L+S), \hat{t}_{\gamma_{i-1}}^{\mathcal{O}}(L), \hat{t}_{\gamma_{i-1}}^{\mathcal{O}}(L+S)$ can still be estimated by following 14 .

\section{Numerical Results}

The performance of the proposed decentralized autonomous intersection management method is evaluated in this section. The parameters of the intersection are $L=$ $150 \mathrm{~m}$ and $S=10 \mathrm{~m}$, with a sampling interval $\Delta s=2 \mathrm{~m}$. For illustrative and comparative purposes, let us consider a case with $20 \mathrm{CAVs}$ all of which are assumed to leave the intersection (the MZ) at the same terminal speed $\bar{v}=10 \mathrm{~m} / \mathrm{s}$. Without loss of generality, the control problem is initialized with randomized initial conditions $v_{i}(0)$ and $t_{i}(0)$ for all CAVs subject to the constraints imposed in Assumptions 2 and 3 In particular, the initial speeds of all CAVs follow a uniform distribution within $\left[v_{\min }, v_{\max }\right]$, while their arrival times $t_{i}(0)$ follow a Poisson distribution. Moreover, the entry of each CAV is also randomly generated. The convex DMPC is solved by using YALMIP and MOSEK [17] in Matlab on a PC with Intel Core i5 $2.9 \mathrm{GHz}$ and $8 \mathrm{~GB}$ of RAM.

The optimality of DMPC solutions is investigated by studying the trade-off between travel time and energy consumption. In terms of the comparison, various prediction horizon lengths $N_{p}=\{10,15,20\}$ are used and the solution of the centralized convex optimal control problem (4) proposed in [11] is also included. The results shown in Fig. 5

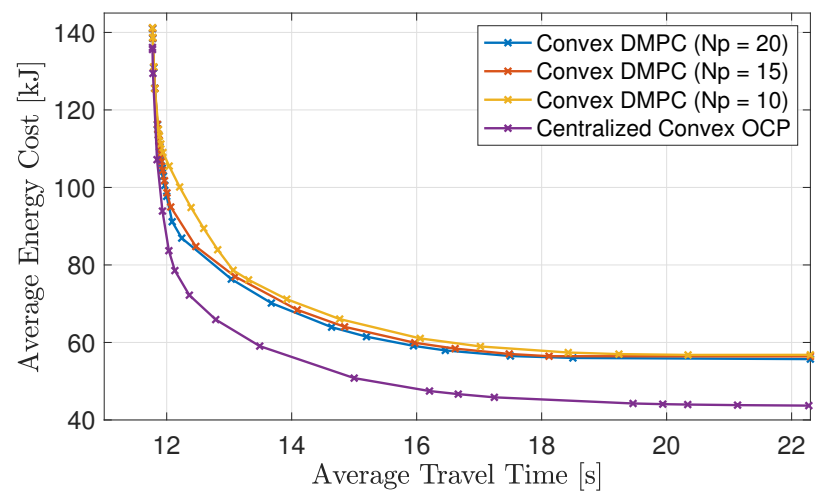

Fig. 5. The trade-offs between average battery energy consumption and average travel time at the arrival rate of $500 \mathrm{veh} / \mathrm{h}$ per lane for the DMPC with varied prediction horizon length and the centralized method [11].

point out for both centralized and decentralized methods the importance of examining the energy-time trade-off, as a small change in the travel time can significantly affect the energy efficiency. For example, when the decentralized method is used with $N_{p}=10$, an increase in travel time of approximately $30 \%$ can lead to a roughly $56 \%$ reduction in the energy consumption, while further increase in travel time can eventually yield up to $60 \%$ energy consumption reduction. The comparison among the three prediction horizon lengths indicates that the overall optimality increases as the $N_{p}$ increases. The reason is that increasing the horizon tends to enhance the ability to anticipate the future behavior of each CAV to satisfy the collision and FIFO constraints, at a price of higher computational burden. However, the improvement in terms of energy consumption optimality from $N_{p}=10$ to 20 is less than $4 \%$ for most cases, and therefore, the subsequent studies in this paper adopt $N_{p}=10$ for the DMPC problem.

Moreover, by comparing with the centralized OCP, it can be expected that the Pareto frontiers of the DMPC are always above (less optimal) that of the centralized method. When $W_{2} \gg W_{1}$, the optimality of the decentralized approach is close the that of the centralized approach. This can be understood as when the energy cost becomes negligible in the objective function, the driving speed solved by both methods is pushed to the upper limit to minimize the travel time, resulting in a similar energy cost. As the weight $W_{1}$ for the energy cost is gradually increased, the optimality of the decentralized method deteriorates as compared to the solution of the centralized method, resulting in a maximum gap of $22 \%$ between the two methods when the average travel time is $12.75 \mathrm{~s}$, and after this time resulting in a gap of no less than $21 \%$. The reason is that when the travel time is relaxed, there exists more room for speed optimization, and therefore the centralized method can yield superior solutions in terms of energy consumption.

To gain more insight into the behavior of both the centralized and decentralized control schemes, Fig. 6 shows the traveled distance of all $20 \mathrm{CAVs}$ by solving the DMPC problem (17) subject to an average travel time $12.75 \mathrm{~s}$ at an arrival rate of $500 \mathrm{veh} / \mathrm{h}$ per lane. It can be observed that all CAVs obey the FIFO policy without experiencing rear-end and side collisions throughout the simulation, and therefore, the validity of the solutions of the DMPC problem (17) is verified.

The optimal speed trajectories (with respect to time) in the decentralized and centralized cases at the same average travel time $12.75 \mathrm{~s}$ are shown in Figs. 7) and 8, respectively, where the speed profiles in both scenarios are grouped based on the heading directions for illustration purposes. Unlike the centralized method where all CAVs decelerate immediately after reaching the peak speeds, the CAVs in the decentralized framework tend to cruise at the peak speed and apply more intensive braking when approaching the exit of the $\mathrm{MZ}$ as compared to the centralized solution. Mechanical braking is engaged on this occasion to meet to the sharp deceleration, which due to the power dissipation is not optimal in terms of energy efficiency. 


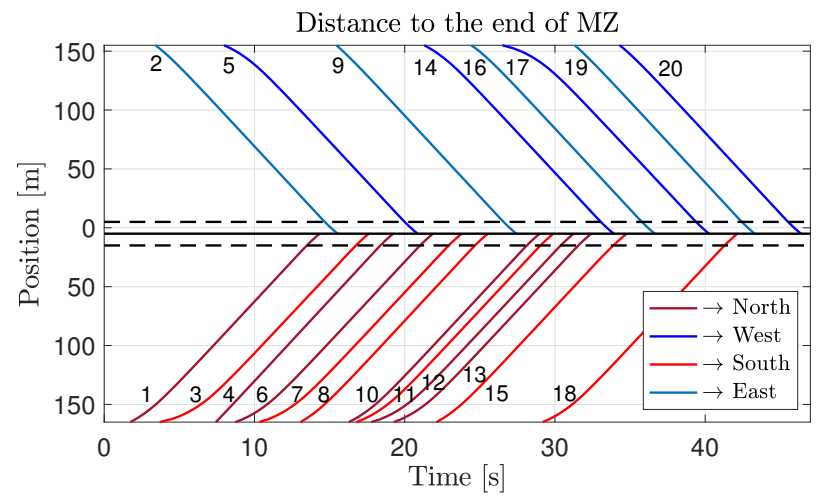

Fig. 6. Traveled distance trajectories (distance to the end of MZ) by solving the DMPC (17) subject to an average travel time $12.75 \mathrm{~s}$ at an arrival rate of $500 \mathrm{veh} / \mathrm{h}$ per lane. The horizontal dashed lines correspond to the entry of the MZ, while the horizontal continuous black line represents the end of the MZ. The four vehicle heading directions are denoted using different colors.

The DMPC computational time of a single CAV $i$ at every step with the sampling interval $\Delta s=2 \mathrm{~m}$ is shown in Fig. 9 . The dashed line denotes the estimated permissible computational time at every interval distance $\Delta s$ in the space domain, obtained by $\Delta s / v_{i}(k)$. As it can be seen, the computational time of vehicle $i$ is strictly below the maximum allowed time, which validates the implementation of the DMPC approach. A further increase on $\Delta s$ could increase the computational capability of the DMPC in coping with additional uncertainties, such as communication delay, at a price of computational precision.
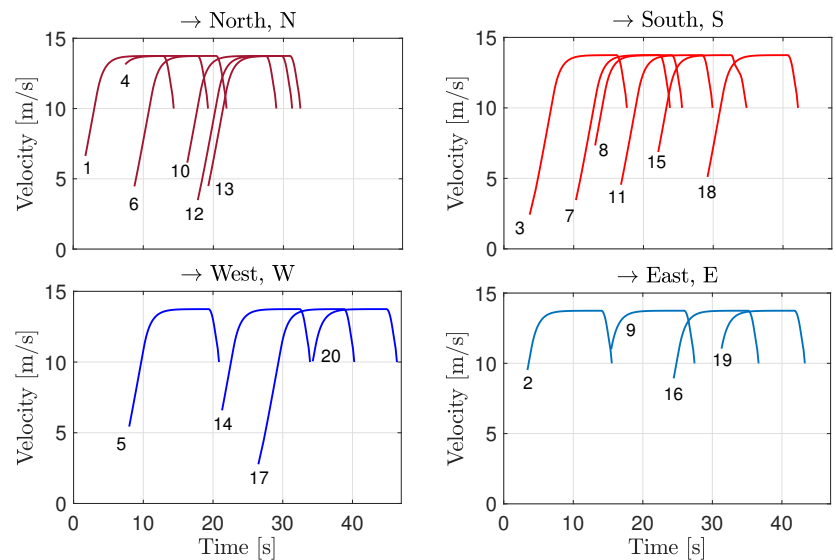

Fig. 7. Optimal speed profiles by solving the DMPC problem (17) subject to an average travel time $12.75 \mathrm{~s}$ for all CAVs at an arrival rate of 500 $\mathrm{veh} / \mathrm{h}$ per lane.

\section{CONCLUSIONS}

In this paper, the problem of optimally controlling batteryelectric CAVs crossing an unsignalized intersection is addressed in a decentralized manner. A DMPC-based control strategy is proposed to sequentially minimize the electric energy consumption and the travel time of all CAVs. With the convex modeling technique and MPC framework, a unique optimal solution can be guaranteed with a high computational efficiency. The numerical examples examine
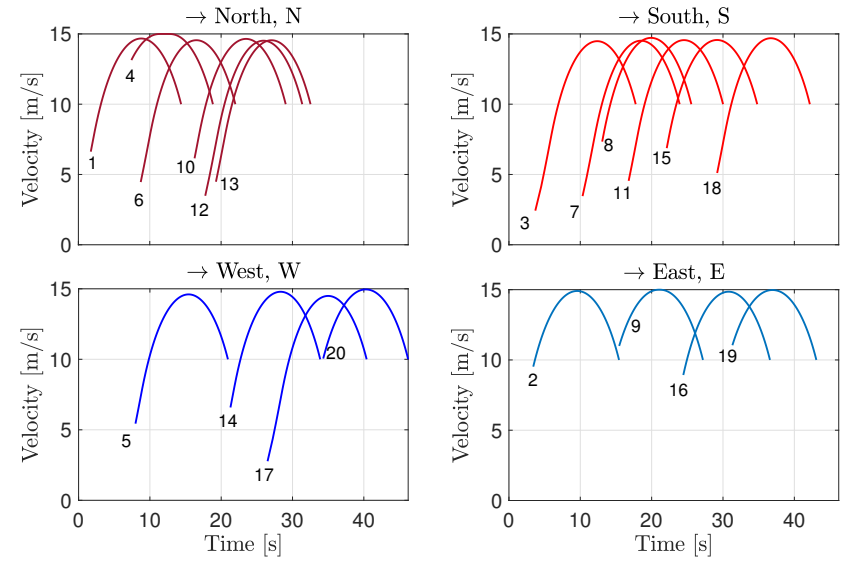

Fig. 8. Optimal speed profiles by solving the centralized problem 4 subject to an average travel time $12.75 \mathrm{~s}$ for all CAVs at an arrival rate of $500 \mathrm{veh} / \mathrm{h}$ per lane

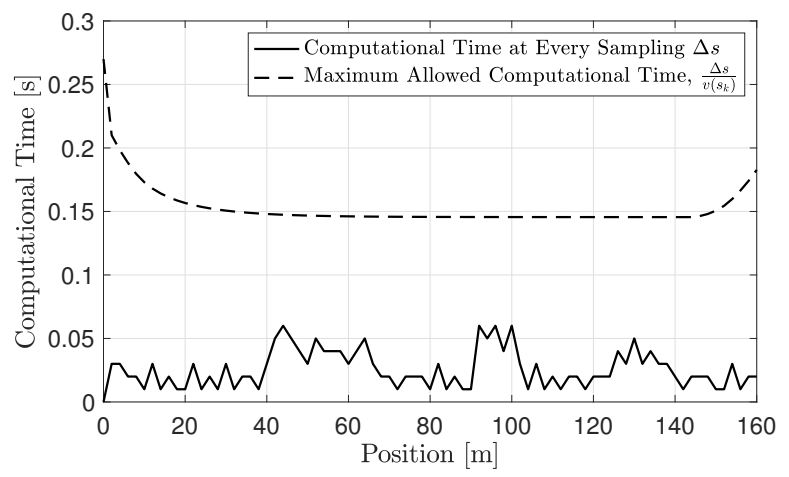

Fig. 9. Computational time of an example CAV $i$ and the corresponding maximum allowed computational time $\Delta s / v_{i}(k)$ with sampling $\Delta s=2 \mathrm{~m}$ by solving the DMPC problem.

the trade-off between energy consumption and travel time for different prediction horizon lengths. The proposed decentralized method is also benchmarked against a centralized optimal control method. Finally, the computation efficiency of the DMPC is examined and the results show the practical potential of the proposed scheme. Future work will focus on the development of robust DMPC methods to cope with communication, measurement and model uncertainties.

\section{REFERENCES}

[1] L. Chen and C. Englund, "Cooperative intersection management: A survey," IEEE Transactions on Intelligent Transportation Systems, vol. 17, no. 2, p. 570586, 2016.

[2] Y. Bian, Y. Zheng, W. Ren, S. E. Li, J. Wang, and K. Li, "Reducing time headway for platooning of connected vehicles via V2V communication," Transportation Research Part C: Emerging Technologies, vol. 102, pp. 87-105, 2019.

[3] G. Velasco-Hernandez, E. Caicedo-Bravo, J. Barry, and J. Walsh, "Intersection management systems and internet of things: A review," in Proc. of 2020 IEEE 16th International Conference on Intelligent Computer Communication and Processing (ICCP). IEEE, 2020, pp. 439-445.

[4] E. Namazi, J. Li, and C. Lu, "Intelligent intersection management systems considering autonomous vehicles: A systematic literature review," IEEE Access, vol. 7, pp. 91 946-91 965, 2019.

[5] J. Rios-Torres and A. A. Malikopoulos, "A survey on the coordination of connected and automated vehicles at intersections and merging at highway on-ramps," IEEE Transactions on Intelligent Transportation Systems, vol. 18, no. 5, pp. 1066-1077, 2017. 
[6] N. Murgovski, G. R. de Campos, and J. Sjoberg, "Convex modeling of conflict resolution at traffic intersections," in Proc. of IEEE 54th Annual Conference on Decision and Control, 2015, pp. 4708-4713.

[7] S. A. Fayazi and A. Vahidi, "Mixed-integer linear programming for optimal scheduling of autonomous vehicle intersection crossing," IEEE Transactions on Intelligent Vehicles, vol. 3, no. 3, pp. 287-299, 2018.

[8] L. Riegger, M. Carlander, N. Lidander, N. Murgovski, and J. Sjoberg, "Centralized MPC for autonomous intersection crossing," in Proc. of International Conference on Intelligent Transportation Systems (ITSC), 2016, pp. 1372-1377.

[9] A. Hadjigeorgiou and S. Timotheou, "Optimizing the trade-off between fuel consumption and travel time in an unsignalized autonomous intersection crossing," in Proc. of 2019 IEEE Intelligent Transportation Systems Conference (ITSC). IEEE, 2019, pp. 2443-2448.

[10] B. Chen, X. Pan, S. A. Evangelou, and S. Timotheou, "Optimal control for connected and autonomous vehicles at signal-free intersections," in Proc. of IFAC World Congress, 2020.

[11] X. Pan, B. Chen, S. A. Evangelou, and S. Timotheou, "Optimal motion control for connected and automated electric vehicles at signal-free intersections," in Proc. of 2020 59th IEEE Conference on Decision and Control (CDC). IEEE, 2020, pp. 2831-2836.

[12] G. R. D. Campos, P. Falcone, R. Hult, H. Wymeersch, and J. Sjoberg, "Traffic coordination at road intersections: Autonomous decisionmaking algorithms using model-based heuristics," IEEE Intelligent Transportation Systems Magazine, vol. 9, no. 1, p. 821, 2017.

[13] A. A. Malikopoulos, C. G. Cassandras, and Y. J. Zhang, "A decentralized energy-optimal control framework for connected automated vehicles at signal-free intersections," Automatica, vol. 93, pp. 244256, 2018.

[14] Y. Wu, H. Chen, and F. Zhu, "DCL-AIM: Decentralized coordination learning of autonomous intersection management for connected and automated vehicles," Transportation Research Part C: Emerging Technologies, vol. 103, p. 246260, 2019.

[15] K. Zhang, D. Zhang, A. de La Fortelle, X. Wu, and J. Gregoire, "State-driven priority scheduling mechanisms for driverless vehicles approaching intersections," IEEE Transactions on Intelligent Transportation Systems, vol. 16, no. 5, pp. 2487-2500, 2015.

[16] A. P. Chouhan and G. Banda, "Autonomous intersection management: A heuristic approach," IEEE Access, vol. 6, pp. 53287-53295, 2018.

[17] J. Löfberg, "Yalmip : A toolbox for modeling and optimization in matlab," in Proc. of the CACSD Conference, Taipei, Taiwan, 2004. 